\title{
Preparatory Delay Activity in the Monkey Parietal Reach Region Predicts Reach Reaction Times
}

\author{
Lawrence H. Snyder, ${ }^{1}$ Anthony R. Dickinson, ${ }^{1}$ and Jeffrey L. Calton ${ }^{2}$ \\ ${ }^{1}$ Department of Anatomy and Neurobiology, Washington University School of Medicine, St. Louis, Missouri 63110, and 2Department of Psychology, \\ California State University, Sacramento, California 95819
}

To acquire something that we see, visual spatial information must ultimately result in the activation of the appropriate set of muscles. This sensory to motor transformation requires an interaction between information coding target location and information coding which effector will be moved. Activity in the monkey parietal reach region (PRR) reflects both spatial information and the effector (arm or eye) that will be used in an upcoming reach or saccade task. To further elucidate the functional role of PRR in visually guided movement tasks and to obtain evidence that PRR signals are used to drive arm movements, we tested the hypothesis that increased neuronal activity during a preparatory delay period would lead to faster reach reaction times but would not be correlated with saccade reaction times. This proved to be the case only when the type of movement and not the spatial goal of that movement was known in advance. The correlation was strongest in cells that showed significantly more activity on arm reach compared with saccade trials. No significant correlations were found during delay periods in which spatial information was provided in advance. These data support the idea that PRR constitutes a bottleneck in the processing of spatial information for an upcoming arm reach. The lack of a correlation with saccadic reaction time also supports the idea that PRR processing is effector specific, that is, it is involved in specifying targets for arm movements but not targets for eye movements.

Key words: monkey; cortex; arm movement; reaction time; motor intention; preparatory set; parietal

\section{Introduction}

Spatial and effector information interact in frontal areas such as the supplemental eye fields (Fujii et al., 2002) and premotor cortex (Hoshi and Tanji, 2004) as well as in posterior parietal cortex (Snyder et al., 1997). Cells in the parietal reach region (PRR) and the lateral intraparietal area (LIP) are driven when either spatial or effector information is presented in isolation (Calton et al., 2002; Dickinson et al., 2003). Many PRR neurons are activated when an animal prepares a reach but not a saccade, whereas many LIP neurons show the reverse pattern. This is consistent with an effector-specific functional role in visually guided movement. Here we extend these findings by showing a trial-by-trial correlation between PRR activity and movement reaction time (RT) (Riehle and Requin, 1993).

Neuronal signals that are correlated with an upcoming action or motor plan are often called "preparatory set" or "motor set" (Tanji and Evarts, 1976; Weinrich et al., 1984). Set signals occur throughout frontal, premotor, and motor cortex, superior colliculus, and basal ganglia (Lecas et al., 1986; Alexander and Crutcher, 1990; Jaeger et al., 1993; Kurata, 1993; Riehle and

\footnotetext{
Received Feb. 3, 2006; revised Aug. 9, 2006; accepted Aug. 10, 2006.

This work was supported by the National Institutes of Health (L.H.S., J.L.C.) and the McDonnell Foundation (A.R.D.).

Correspondence should be addressed to Lawrence H. Snyder, Department of Anatomy and Neurobiology, Box 8108, Washington University School of Medicine, 660 South Euclid Avenue, St. Louis, M0 63110. E-mail: larry@eye-hand.wustl.edu.

DOI:10.1523/JNEUROSCI.0513-06.2006

Copyright $\odot 2006$ Society for Neuroscience $\quad$ 0270-6474/06/2610091-09\$15.00/0
}

Requin, 1993; Hanes et al., 1995; Basso and Wurtz, 1997, 1998; Dorris et al., 1997; Dorris and Munoz, 1998; Everling et al., 1999; Crammond and Kalaska, 2000; Everling and Munoz, 2000; Connolly et al., 2002, 2005; DeSouza et al., 2003; Lee and Assad, 2003). These signals may reflect advance preparation for particular actions, which in turn affects RT and/or accuracy, even in nonhuman primates (Stoet and Snyder, 2003; Churchland et al., 2006). Trial-by-trial correlations of activity with RT have been observed in the primary motor cortex and basal ganglia with reaching (Lecas et al., 1986; Lee and Assad, 2003) and in the frontal eye fields and superior colliculus with saccades (Hanes and Schall, 1996; Dorris et al., 1997; Everling and Munoz, 2000). These correlations can provide evidence for a causal role in movement initiation.

Set signals are not necessarily correlated with movement RT. A high-level working memory signal (Baddeley, 2003), for example, may reflect an upcoming plan for action but not be involved in initiating movement. There are only a few reports of trial-bytrial correlation of parietal activity with reach or saccade RT (Requin et al., 1990; Roitman and Shadlen, 2002; Cohen et al., 2004) (see also Elkington et al., 1992). Spatial signals in posterior parietal cortex may reflect spatial attention or salience, not motor set (Bushnell et al., 1981; Corbetta et al., 1993; Constantinidis and Steinmetz, 2001; Corbetta and Shulman, 2002; Bisley and Goldberg, 2003). Even the effector-specific signals in PRR and LIP, described above, may have only an indirect relationship with movement initiation and consequently are often referred to as "motor intention" rather than motor set (Andersen, 1995; Sny- 
der et al., 1997; Thoenissen et al., 2002; Connolly et al., 2003; Sirigu et al., 2004).

In the current study, we tested for correlations between preparatory delay activity and movement reaction times. We predicted and confirmed that correlations would occur in PRR but not in neighboring area 5 and with reach RT but not saccade RT. We further predicted that correlations would be strongest when both spatial and effector information were known compared with when only spatial or only effector information was known. To our surprise, this prediction was not met. Instead, correlations were highest when only effector information was known.

\section{Materials and Methods}

Two rhesus monkeys (M1 and M2) performed rapid eye movements and forelimb reaches to peripheral visual targets. All procedures conformed to the Guide for the Care and Use of Laboratory Animals and were approved by the Washington University Institutional Animal Care and Use Committee. Each animal was prepared for experiments by attaching a stabilization platform to the head and implanting a scleral search coil under the conjunctiva of one eye (Judge et al., 1980). Surgery was performed using isoflurane anesthesia (1-2\%).

For experiments, the animal sat in a custom-designed monkey chair (Crist Instruments, Hagerstown, MD) that allowed a wide range of arm movement. Visual stimuli were projected (ECP 3000; Electrohome, Kitchener, Ontario, Canada) onto a $43 \mathrm{~cm}$ vertically oriented touch screen (Keytec, Garland, TX) $25 \mathrm{~cm}$ from the animal. Spatial resolution for touches was $0.1 \mathrm{~mm}$, and temporal resolution was either 30 or $8 \mathrm{~ms}$ (the latter achieved using a custom touch-screen controller). Eye position was recorded with $0.05^{\circ}$ precision every $2 \mathrm{~ms}$ (CNC Engineering, Seattle, WA).

Animals performed three different paradigms (see Fig. 1A). In standard memory saccades and reaches, every trial began with the monkey foveating and reaching toward a blue central square in an otherwise dark room. After $500 \mathrm{~ms}$, a peripheral target appeared for $300 \mathrm{~ms}$. A red target instructed a saccade, a green target instructed a reach (color assignment was reversed in the second animal), and a white target instructed a combined reach plus saccade. The target could appear in the center of the response field of the neuron being recorded or on the opposite side of the fovea. Additional target locations were used in a subset of cells, but those data are not reported here. After an 800 ms delay period, the fixation target was blanked, cueing the animal to initiate the instructed movement to the remembered target location. A liquid reward was delivered after $300 \mathrm{~ms}$ of peripheral fixation or reach.

The second type of paradigm, the cue-delay-target task, also began with foveation and touch of a blue central square. After 500-800 ms, the fixation point changed color to instruct an arm movement, eye movement, or a combined eye and arm movement (see cell counts in Table 3). The central color cue remained present for the remainder of the trial. After a delay of 600, 900, or $1200 \mathrm{~ms}$, a blue peripheral target appeared inside or outside the response field. Animals were free to acquire the peripheral target as soon as it appeared, as per the previous movementtype instruction. After holding the target for $400 \mathrm{~ms}$, they received a liquid reward.

The third type of paradigm, the target-delay-cue task, was similar to the cue-delay-target task, except that the spatial target was presented first and the foveal color change occurred second. The variable delay period was similar to that of cue-delay-target trials, and the animal was free to acquire the target once the foveal color change had occurred. Target-delay-cue and cue-delay-target trials were interleaved for $>90 \%$ of recorded cells. Standard memory trials were obtained in a subset of cells in separate blocks. Within each block, saccade trials, reach trials, and combined saccade plus reach trials were fully interleaved.

Targets were $1.6 \times 1.6^{\circ}$ squares. Animals were required to fixate and touch within 3 and $6^{\circ}$, respectively, of visible targets and within 8.5 and $7.5^{\circ}$ of remembered targets. Within $200 \mathrm{~ms}$ of acquisition, these windows were shrunk to $2-3^{\circ}$, centered about the location of the initial touch. A trial was terminated, and the data were not analyzed if the eyes or arm left the window prematurely, if the eyes moved before the reward was deliv- ered on a reach trial, if the arm moved before the reward was delivered on a saccade trial, or if the reaction time was extremely slow (typically $>1 \mathrm{~s}$, although this value was adjusted per animal and task). Errors were punished with a $1-2 \mathrm{~s}$ timeout. Success rates were typically $>80 \%$.

Recording procedures. Recordings were made from three hemispheres of two adult rhesus monkeys. Recording chambers were centered at 5-7 $\mathrm{mm}$ posterior and $12 \mathrm{~mm}$ lateral (Horsley-Clarke coordinates) and placed flush to the skull. Signals from electrodes with impedance measured at $1 \mathrm{kHz}$ of $0.3-2.5 \mathrm{M} \Omega$ (Frederick Haer Company, Bowdoinham, $\mathrm{ME}$ ) were amplified and filtered (bandpass, $\sim 500-5000 \mathrm{~Hz}$ ), and single units were isolated using a dual-window discriminator (Bak, Germantown, MD). Spike times were recorded with $1 \mathrm{~ms}$ precision.

While searching for cells, animals performed nondelayed, center-out combined eye and arm movements to $20^{\circ}$ peripheral targets located in each of eight equally spaced directions. Data were then collected in the cue-delay-target, target-delay-cue, and sometimes the standard memory task from any cell that changed firing rate at any point in the search task. Each trial type was repeated $8-10$ times for a total of $\sim 180$ trials per cell (3 paradigms $\times 2-3$ movement types $\times 2$ targets $\times 8-10$ repetitions). Data from cells that were not modulated in any paradigm were excluded from additional analysis ( $<10 \%$ of recorded cells).

Our delineation of PRR has been described previously (Calton et al., 2002). Cortical regions $1-4 \mathrm{~mm}$ anterior to the anterior boundaries of PRR and LIP (Dickinson et al., 2003) were defined as area 5. Anatomical magnetic resonance images were acquired to confirm these localizations and are described in the previously cited publications.

Data analysis. Data were analyzed to determine whether movement RT was related to delay period activity. RT was measured from the end of the delay period (depending on the paradigm, fixation point offset, or the appearance of the spatial target or movement cue) to the onset of movement. Trials with very short RTs $(<130 \mathrm{~ms})$ or very long RTs $(>300 \mathrm{~ms}$ for saccades, $>800 \mathrm{~ms}$ for reaches) were eliminated. In the standard memory paradigm, the memory period duration was fixed and therefore predictable. For this paradigm, a minimum RT of $20 \mathrm{~ms}$ was used. Changes in these criteria had minimal effects on the results. Datasets containing fewer than three observations were excluded. Similar results were obtained using a minimum of five data points. Data from $<10 \%$ of cells were excluded as a result of these criteria.

Both RT and 1/RT were tested for a relationship with firing rate (Reddi et al., 2003). We report data using RT. However, nearly identical results were obtained using 1/RT.

Both type I and type II regressions were performed. Type I regressions assume that the variable on the $x$-axis is chosen by the investigator on each trial and therefore does not contribute any variance to the relationship, whereas type II regressions assume that both variables are experimentally determined and contribute equal amounts of variance to the relationship. Similar results were obtained in each case.

PRR cells were divided into visual, visuomovement, and movement cells based on responses in the standard memory reach task, by analogy to a similar classification of frontal eye field cells based on the standard memory saccade task (Bruce and Goldberg, 1985). A "visual" response was calculated as the increase in firing in the interval 50-300 ms after target appearance compared with $200-400 \mathrm{~ms}$ before target appearance. A "movement" response was calculated as the increase in firing in the 100 $\mathrm{ms}$ before the onset of arm movement compared with activity 200-300 $\mathrm{ms}$ before movement onset. A contrast ratio of movement to visual responses was then used to classify cells, using criterion values of -0.4 to divide visual from visuomovement cells and 0.4 to divide visuomovement from movement cells. Changes in these arbitrary criteria had minimal effect on our results.

\section{Results}

\section{Behavior}

Reaction times were measured in three different delayed saccade and reach paradigms: a standard memory task, the cue-delaytarget task, and the target-delay-cue task (Fig. 1A). Table 1 shows median RTs for the two animals used in this study. The fastest responses typically occurred in the standard memory task. 
A
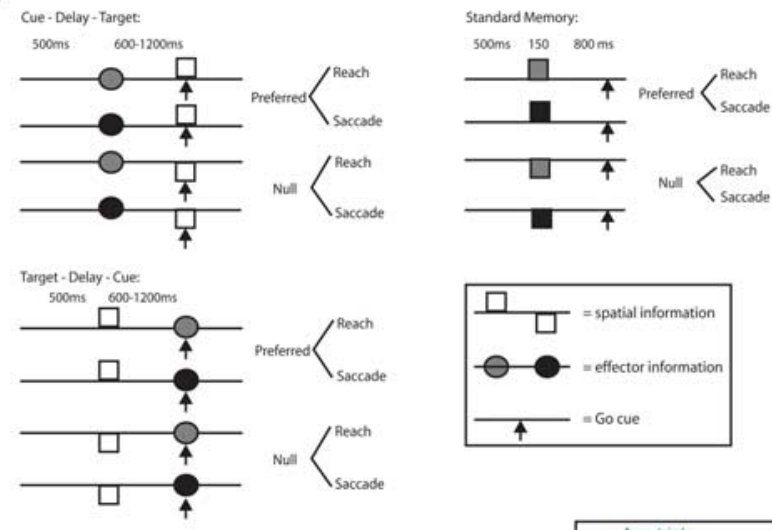

B PRR

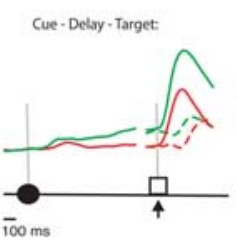
Target-Delay-Cue:
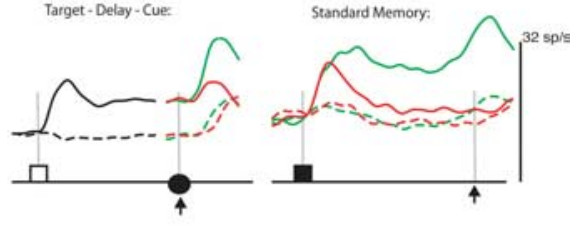

C Area 5
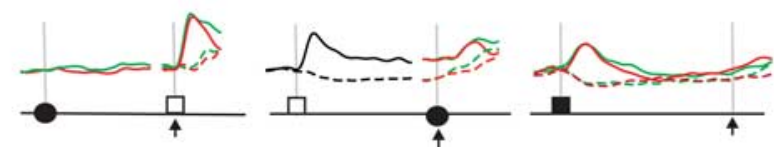

Figure 1. Three delayed saccade and reach paradigms. $\boldsymbol{A}$, Each time line shows when effector information (reach or saccade; shaded circle) and spatial information (preferred or null direction; open square) are provided and when the animal becomes free to move (arrow). In the cue-delay-target task, animals prepare a reach or a saccade and wait to receive information about where to move. In the target- delay-cue task, animals know where to move but wait to learn the type of movement. In the standard memory task, animals know both movement type and destination and await only the "go" cue. For additional details, see Results. B, Populationaveraged activity in PRR in each of the three tasks. C, Population-averaged activity in area 5 in each of the three tasks.

In this task, animals knew both the type of movement (reach or saccade) and the goal of the movement (the spatial location of the target) and initiated movements in response to fixation offset. The next fastest times occurred in the cue-delay-target task. In this task, animals knew the movement type in advance and initiated movements in response to a peripheral flash that signaled the target location. A peripheral flash evokes automatic orienting and thereby facilitates a rapid response (Yantis and Jonides, 1984). The slowest times occurred in the target-delay-cue task. In this task, animals knew only the spatial location in advance and initiated movements in response to a color change at the fovea that signaled which effector was to be used. The slow responses may reflect the fact that the perception and processing of an endogenous (symbolic) foveal color cue is likely to be slow compared with the perception and processing of an exogenous peripheral cue.

Three delay period durations $(0.6,0.9$, and $1.2 \mathrm{~s})$ were interleaved in the cue-delay-target and target-delay-cue tasks. This variability may also contribute to the longer RTs in these tasks compared with the standard memory task, which was performed with a single delay period $(0.8 \mathrm{~s})$. In cue-delay-target and targetdelay-cue tasks, shorter delay periods were associated with longer RTs. For saccades, the average RT was $7 \mathrm{~ms}$ longer after a $0.6 \mathrm{~s}$ delay period compared with a $1.2 \mathrm{~s}$ delay period. For reaches, this difference was $32 \mathrm{~ms}$.
The direction of movement strongly influenced saccade and reach RT, with median values varying by as much as $35 \mathrm{~ms}$ across different directions. These effects were consistent across sessions and were very large compared with the measured standard error. In contrast, the effect of moving the eyes and arm together to the same target, compared with moving them in isolation, had only a small effect on RT, delaying saccades by $1 \mathrm{~ms}$ and delaying reaches by $4 \mathrm{~ms}$ (median values) (for details, see Snyder et al., 2002).

\section{Neural activity}

We tested the hypothesis that PRR delay period activity reflects effector-specific motor preparation by determining whether activity was correlated with reach reaction times. Activity was recorded during standard memory, cue-delay-target, and targetdelay-cue tasks. As a control for the specificity of the correlation, we also tested area 5 neurons outside of PRR.

Figure $1 B$ shows the population-averaged activity recorded in the three tasks. In all three tasks, neurons respond vigorously around the time of a reaching movement and weakly or not at all around the time of a saccade (far right of each panel; green vs red traces). Before movement in the cue-delay-target task (left panel), instructing only the effector type (but not the spatial goal) resulted in increased delay period activity on reach but not saccade trials (green vs red trace) (for details, see Calton et al., 2002; Dickinson et al., 2003). Before movement in the target-delaycue task (middle panel), instructing only the spatial goal of the movement (and not the effector type) resulted in increased delay period activity when the target was in the preferred direction (by definition) but no increase in firing when the target was in the opposite, or null, direction (again, by definition; solid vs dashed line). Finally, in the standard memory task (right panel), instructing both the spatial goal and the effector type resulted in a large sustained increase in delay period activity for reaches in the preferred direction, a transient increase followed by a small sustained increase for saccades in the preferred direction, and no increase for reaches or saccades in the null direction (Snyder et al., 1997).

Figure $1 C$ shows similar population-averaged activity recorded in area 5 . This population does not cover all of area 5 but only those cells found anterior to area LIP, on the medial bank of the intraparietal sulcus, distal to PRR and to the medial intraparietal area. Prominent activations occurred during and immediately after target presentation (e.g., at the start of the standard memory and target-delay-cue tasks and at the end of the cuedelay-target task). Only small activations occurred during eye or arm movements (e.g., at the end of the standard memory task and target-delay-cue task). In contrast to the PRR neurons (Fig. $1 B$ ), area 5 neurons showed almost no effector-specific activity. This was true of both delay and movement periods.

It seemed reasonable that, across tasks, reach reaction time might be correlated with the strength of delay period activity. In fact, this was not the case for either PRR or area 5 (Table 2). For example, the reach tasks associated with the fastest and slowest RT (memory task and target-delay-cue tasks, respectively) were both associated with the highest level of delay period firing [17.6 spikes per second (sp/s)]. Perhaps cortical regions play somewhat different roles during different tasks. In this case, correlations might be found within the trials of a particular task.

\section{Cue-delay-target task, PRR example cell}

Individual reach, saccade, and coordinated reach plus saccade trials are illustrated in $B-D$, respectively, of Figure 2. Movements in the preferred direction are on shown on the left of each panel 
(filled histograms), and movements in the null direction are shown on the right (open histograms). Data are aligned on target presentation (narrow gray line). Reach and saccade reaction times can be inferred from the abrupt deflections in eye and arm position (bottom of panels) that follow shortly after target presentation. We asked whether these reaction times were systematically related to the number of spikes in the $300 \mathrm{~ms}$ interval immediately before target presentation.

The preparatory delay period activity of PRR neurons was clearly related to RT in the cue-delay-target task. Figure $2 \mathrm{~A}$ shows trial-by-trial reaction times as a function of firing rate in the $300 \mathrm{~ms}$ interval before the target appearance, obtained from a single PRR neuron. Reach RTs (left subpanel) were generally longer after delay periods in which fewer spikes were recorded. The correlation between firing rate and reach reaction time (see Materials and Methods) was significant ( $p=0.03)$, with a Pearson's correlation coefficient $(r)$ of -0.40 .

The relationship between RT and firing rate could reflect a causal role for PRR in initiating a reach. However, an alternative possibility is that the relationship reflects an indirect, nonspecific effect. For example, an increased state of alertness might lead to both increased activity and shorter reaction times. An effector nonspecific relationship can be ruled out by considering the relationship between activity and saccadic RT (Fig. 2A, right subpanel). The correlation coefficient for the pooled saccade data were $+0.09(p=0.8)$. Thus, for this PRR cell, significant correlations between neuronal and behavioral data occurred only for reaches.

Four different conditions of the cuedelay-target task are shown in each of the two subpanels of Figure $2 \mathrm{~A}$. Animals were cued to prepare either an isolated movement (green or red) or a combined eye and arm movement (blue). In addition, targets appeared in either the preferred or null direction for the cell being recorded. Correlations between firing rate and arm RT were large and similar across all four conditions: $r=-0.76$ and $r=-0.65$, respectively, for isolated reaches and coordinated reaches performed in the preferred direction (significance levels, $p=0.02$ and $p=$ $0.07)$; and $r=-0.43$ and $r=-0.39$ for isolated and coordinated reaches in the null direction $(p=0.4$ and $p=0.3$ ). Thus, similar correlations were observed regardless of whether the reach was made into or out of the response field and regardless of whether the reach was made alone or paired with a saccade. In contrast, correlations between firing rate and saccade RT were positive in three of four cases (isolated or coortasks
Table 1. Saccade and reach reaction times as a function of task type

\begin{tabular}{lllll}
\hline & Animal & Memory task (ms) & Cue-delay-target (ms) & Target-delay-cue (ms) \\
\hline Saccade RT & M1 & $177(26) ; n=566$ & $175(22) ; n=3285$ & $235(26) ; n=4124$ \\
& M2 & $176(31) ; n=822$ & $200(34) ; n=3319$ & $238(26) ; n=3992$ \\
Reach RT & M1 & $205(65) ; n=380$ & $270(42) ; n=2625$ & $342(65) ; n=2892$ \\
& M2 & $228(102) ; n=1154$ & $347(64) ; n=2375$ & $422(102) ; n=2922$ \\
\hline
\end{tabular}

The values shown are the mean of the individual medians calculated for each direction of movement, followed by the median SD for a single direction of movement (in parentheses). The number of trials ( $n$ ) reflects the sum of all eight movement directions.

Table 2. Delay period activity (mean \pm SEM) relative to baseline in PRR and in area 5 excluding PRR for the three

\begin{tabular}{|c|c|c|c|c|}
\hline Area & Trial type & Memory task (sp/s) & Cue-delay-target (sp/s) & Target- delay-cue (sp/s) \\
\hline \multirow{4}{*}{$\operatorname{PRR}(n=67)$} & \multirow[t]{2}{*}{ Saccade } & $15.6 \pm 1.6$ & \multirow[t]{2}{*}{$13.3 \pm 1.3$} & $17.6 \pm 1.7$ \\
\hline & & $13.6 \pm 1.2$ & & $14.2 \pm 1.2$ \\
\hline & \multirow[t]{2}{*}{ Reach } & $17.6 \pm 1.8$ & \multirow[t]{2}{*}{$16.2 \pm 1.5$} & \\
\hline & & $14.7 \pm 1.4$ & & \\
\hline \multirow[t]{4}{*}{ Area $5(n=50)$} & \multirow[t]{2}{*}{ Saccade } & $10.4 \pm 1.1$ & \multirow[t]{2}{*}{$10.2 \pm 1.1$} & $10.0 \pm 1.0$ \\
\hline & & $9.5 \pm 1.1$ & & $8.5 \pm 1.0$ \\
\hline & \multirow[t]{2}{*}{ Reach } & $11.4 \pm 1.2$ & \multirow[t]{2}{*}{$11.2 \pm 1.2$} & \\
\hline & & $9.7 \pm 1.1$ & & \\
\hline
\end{tabular}

To maximize comparability across task types, only cells tested using all three paradigms are included. For the memory task (column 3), delay period activity is reported separately for preferred and null direction trials and for saccade and reach trials. For the cue- delay-target task, spatial information is not provided until the end of the delay period, so separate values are provided only for saccade and reach trials. For the target-delay-cue task, effector information is not provided until the end of the delay period, so separate values are provided only for preferred and null direction trials.

\section{A. RT versus firing rate}

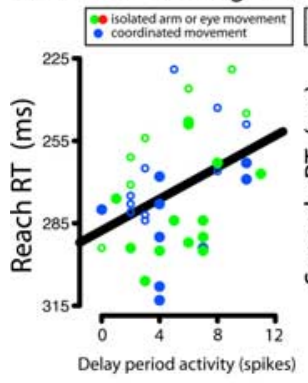

\section{Saccade trials}
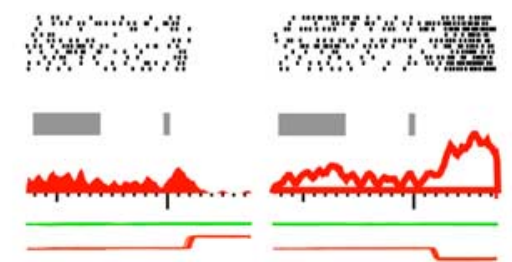

\section{B. Reach trials}
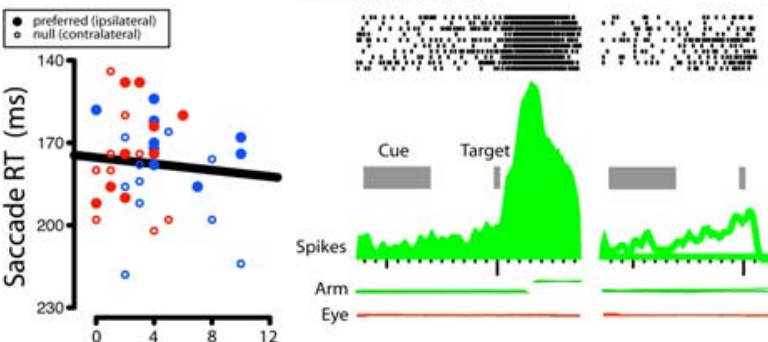
Hin

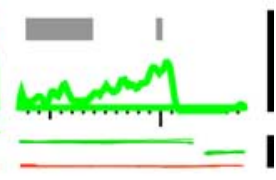

D. Reach + Saccade trials
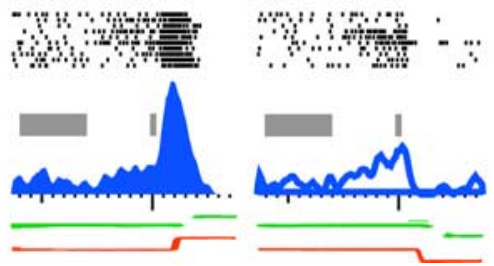

Figure 2. $\quad \boldsymbol{A}$, Data from a single neuron showing a relationship between late delay period activity and reach RT but not saccade RT. Each point reflects a single trial in which an arm movement (left, green), eye movement (right, red), or combined eye and arm movement (both left and right, blue) was directed either into (filled points) or away from (open points) the response field of a neuron. The ordinate (RT) is inverted so that upward slopes correspond to faster responses. $\boldsymbol{B}$, Twenty individual reach cuedelay-target trials that provide the data for two of the green points in $\boldsymbol{A}$. The subpanel on the left (filled histogram) shows 10 trials in the preferred direction, and the subpanel on the right (open histogram) shows 10 trials in the null direction. Data are aligned on the presentation of the spatial target. From top to bottom, rasters represent individual spikes on single trials, histograms of activity with a long gray bar superimposed to show the $900 \mathrm{~ms}$ range of cue presentation times and a short gray bar superimposed to show the instant of spatial target appearance, and horizontal arm and eye position as a function of time, with data from all 10 trials overlaid in each subpanel. Calibration bars: 60 spikes/s (histograms) and $60^{\circ}$ (eye and arm position). C, Twenty individual saccade trials, with the same format as in $\boldsymbol{B}$. $\boldsymbol{D}$, Twenty individual reach plus saccade trials, with the same format as in $\boldsymbol{B}$.

dinated saccades in the preferred direction; coordinated saccades in the null direction).

Correlation coefficients are unitless and therefore do not indicate the magnitude of an effect. We therefore performed a linear regression on the data, asking by how many milliseconds does reaction time change in response to a given change in firing rate. 

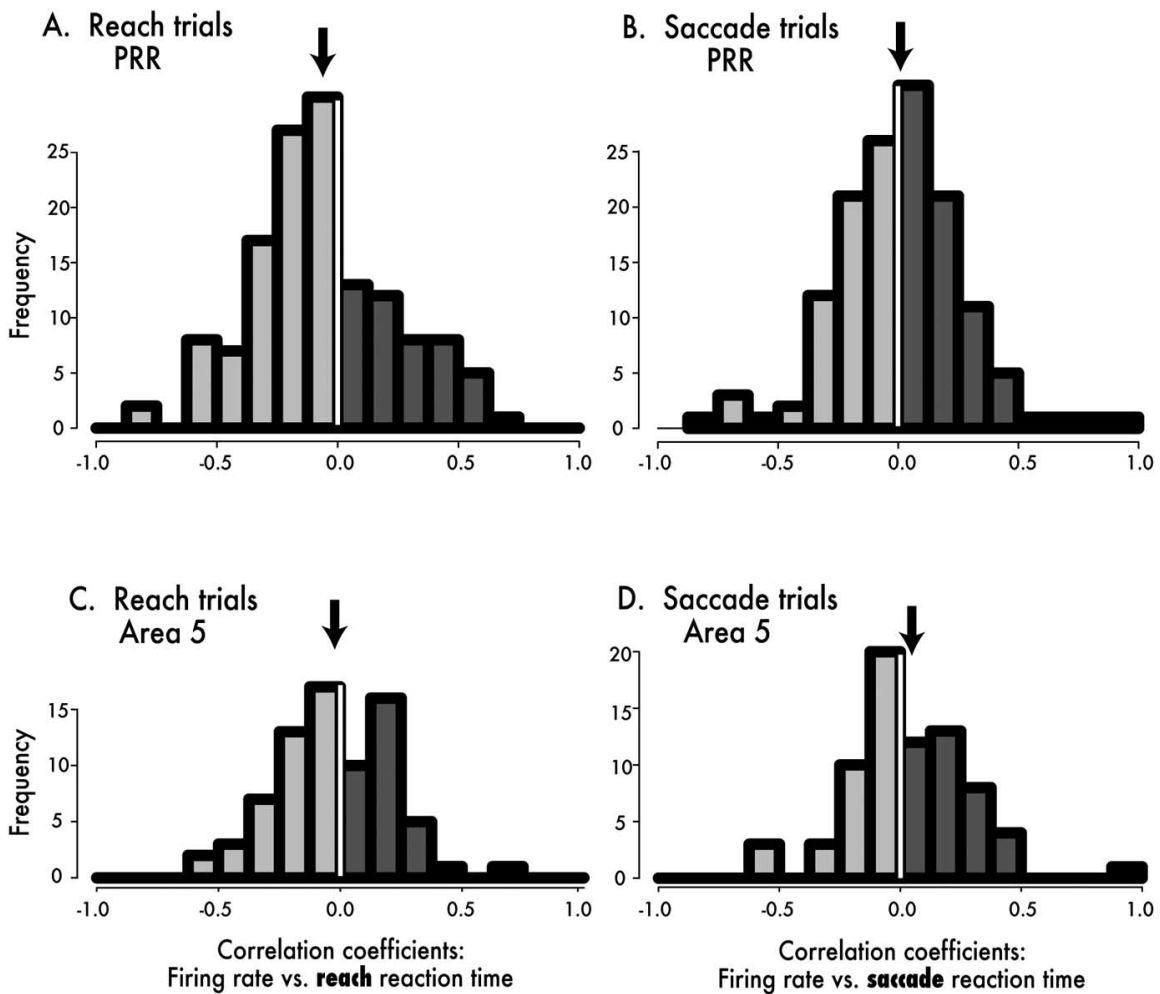

Figure 3. The distribution of regression correlation coefficients between neural activity and reach $R T(A, C)$ or saccade $R T(B, D)$ for PRR (above) and area 5 (below). Activity is measured on cue- delay-target trials in the last $300 \mathrm{~ms}$ of the delay period before the presentation of the spatial target. Negative correlation coefficients, indicating neurons for which higher activity led to faster reaction time, are highlighted by lighter shading. Arrows indicate mean correlation coefficients of $-0.07(p=0.001)$ and 0.00 $(p=0.99)$ for reaches and saccades, respectively, in PRR and $-0.03(p=0.2)$ and $0.04(p=0.3)$ for area 5 .

The effects on isolated and coordinated movements in the preferred and null directions were modeled as sharing an identical slope but different intercepts. For the neuron of Figure 2, this model yields a drop in reach RT of $3.14 \mathrm{~ms}$ for each additional PRR spike occurring within the $300 \mathrm{~ms}$ measurement interval, or $10.5 \mathrm{~ms}$ per $\mathrm{sp} / \mathrm{s}$. In contrast, a regression analysis of the saccade data revealed an increase in saccade RT of $2.1 \mathrm{~ms}$ per sp/s, supporting the idea of an effector-specific effect.

The standard regression model assumes that the values plotted on the abscissa are noise free. In our data, both reaction times and spike counts are observed quantities and therefore subject to noise. A type II regression is more appropriate under these circumstances. This regression, which assumes that noise in the two measurements are equivalent, yields a drop in reach RT of 23.7 $\mathrm{ms}$ per sp/s and an increase in saccade RT of $20.5 \mathrm{~ms}$ per $\mathrm{sp} / \mathrm{s}$. Other regression models [e.g., running separate type I or type II regressions on the data from the different conditions (isolated or coordinated movements, movements in the preferred or null direction); stepwise regression models in which factors that do not contribute significantly to the fit are removed] yielded similar results. Thus, for this cell, there was a significant relationship between delay activity in a cue-delay-target task and reach RT.

\section{Cue-delay-target task, population data}

Across 138 cells recorded in PRR from two animals, many showed a weak relationship between cue-delay-target delay period activity and reach $\mathrm{RT}$. Of 428 observations (138 cells, each tested under two or four different conditions: two directions of isolated reaches, and in some cells, two directions of reaches performed in conjunction with a saccade), faster reach reaction times were significantly correlated with higher activity in 12 cases $(2.8 \%)$. This is not significantly different from what would be expected by chance $(2.5 \%)$ and is also not significantly different from the percentage of neurons showing the reverse correlation $(3.5 \%)$. However, a clear bias toward negative correlations was evident in the distribution of reach-related correlation coefficients across the entire population.

Figure $3 A$ shows the distribution of correlation coefficients across all 138 cells, each tested in four conditions. If there were no significant relationship between delay period activity and reach $\mathrm{RT}$, then the distribution would be centered around 0 . Instead, the average correlation coefficient was -0.07 , which was significantly less than 0 ( $p=0.001$, Wilcoxon's test). A type II regression analysis indicated that the mean magnitude of the effect was a $22.4 \mathrm{~ms}$ drop in reach RT per spike per second ( $p=0.0002$, Wilcoxon's test).

In contrast to the significant relationship between reach RT and delay period activity, there was no relationship between saccadic RT and delay period activity (Fig. $3 B$ ) (mean $r=0.00$, not significantly greater than $0 ; p=0.6$, Wilcoxon's test). A type II regression analysis revealed a mean effect of a $1.9 \mathrm{~ms}$ drop in saccade RT per spike per second ( $p=0.9)$. Analyses based on type I regression produced identical patterns of significance, although the effect size was lower [3.7 $\mathrm{ms}$ drop in reach RT per spike per second ( $p=0.0003$ ) and $1.4 \mathrm{~ms}$ drop in saccadic RT per spike per second $(p=0.9)$ ]. Faster saccade RTs were significantly correlated with higher activity in only 6 of 428 cases $(1.4 \%)$, a value no different than what would be expected by chance $(p=$ 0.2 , proportion test).

A direct comparison of the correlation coefficient distributions confirms that PRR delay period activity has a different relationship with reach compared with saccade RTs (Fig. 3, $A$ vs $B$ ). A positive Kolmogorov-Smirnov test provides evidence that the correlation coefficients are drawn from two different distributions $(D=0.12 ; p<0.01)$, and a $t$ test indicates that the mean correlations differ for reach and saccade trials $(t=2.65$; $\mathrm{df}=815$; $p<0.01)$.

In area 5 , in contrast to PRR, there was no significant relationship between delay period and either reach RT (Fig. 3C: mean $r=$ $-0.03, p=0.2$ ) or saccade RT (Fig. $1 D$ : mean $r=0.04, p=0.3$ ). Of 186 observations in area 5 ( 75 cells tested under two or four conditions), greater activity was significantly correlated with faster reaction times in only $6.4 \%$ of reaches and only $2.7 \%$ of saccades, with neither value different from what would be expected by chance ( $p \geq 0.2$ ). Kolmogorov-Smirnov and $t$ tests fail to indicate that reach and saccade correlation coefficients are drawn from different distributions or have different means ( $p=$ 0.11 and $p=0.09$, respectively).

Because we tested many different task conditions and cortical regions (see below, Target-delay-cue and memory tasks), it is possible that the significant effects observed in PRR using the cue-target paradigm reflect a false-positive finding. We therefore 
analyzed the data from each animal separately to confirm that the effect was present in both animals. The correlation analysis revealed a statistically significant effect in M1 (average correlation coefficient of $-0.09 ; p=0.001 ; 78$ cells) and a trend in M2 (average correlation coefficient of $-0.04 ; p=0.14 ; 60$ cells). A type II regression analysis revealed statistically significant effects in both animal M1 (18.8 ms per sp/s; $p=0.003)$ and M2 $(27.1 \mathrm{~ms}$ per $\mathrm{sp} / \mathrm{s} ; p=0.01)$. The mean correlation and regression coefficients for saccade RTs were not significant, confirming effector specificity [average correlation coefficients of $0.02(p=0.5)$ and $-0.02(p=0.3)$; and average type II regression coefficients of 1.5 $\mathrm{ms}$ per $\mathrm{sp} / \mathrm{s}(p=0.5)$ and $-2.3 \mathrm{~ms}$ per $\mathrm{sp} / \mathrm{s}(p=0.8)$, for monkeys $\mathrm{M} 1$ and $\mathrm{M} 2$, respectively].

Calton et al. (2002) showed that a subset of cells in PRR are significantly more active in the delay period of a cue-delay-target reach task compared with a cue-delay-target saccade task. Of the original 138 cells in the current study, 45 showed reach-specific delay period activity in the cue-delay-target task. These cells had an average correlation coefficient (reach RT vs neuronal activity) of $-0.15(p<0.001)$. In contrast, the 93 cells that were not more active during reach compared with saccade trials had an average correlation coefficient (reach RT vs neuronal activity) of only $-0.03(p=0.2)$. Once again, the significant effects were effector specific; the relationship between saccade RT and firing rate was nonsignificant in both subpopulations of neurons (cells with significant effector-specific modulation during the delay period: $r=$ $0.03, p=0.4$; and those without: $r=-0.01, p=0.5$ ). These data support the idea that effector-specific delay activity in the cuedelay-target trial reflects a preparatory signal that plays a significant role in visually guided reaching.

If it is true that the delay period activity of some PRR cells can influence RT, then we might expect that the cells that show this influence would be those that are most active immediately before the onset of the reach movement. To test this hypothesis, we classified 67 PRR cells into three categories based on their responses during a standard memory reach trial. (Only 67 of the 138 PRR cells were tested using this paradigm.) Visual neurons were strongly activated by a visual stimulus but were at best weakly active immediately before a reach, movement neurons were strongly active before a reach but at best weakly activated by a visual stimulus, and visuomovement neurons were intermediate. We found a correlation between delay activity and reach RT only in the movement neurons $(r=-0.15 ; p=0.001 ; n=16)$. Neither visual nor visuomovement neurons showed a significant effect ( $r=0.00, p=0.9$; and $r=-0.02, p=0.7$, respectively). In area 5 , none of the three categories of cells showed a significant effect $(r=0.02,-0.04$, and -0.02 , respectively; all $p \geq 0.4$ ).

\section{Alternative explanations}

It is conceivable that the delay period activity occurring during cue-delay-target trials could reflect a spatial bias, or guess, regarding where the target will appear. Preparatory set activity recorded in a number of other cortical and subcortical areas is related to a particular movement direction (see Introduction). Alternatively, the activity that occurs during the delay period might be unrelated to spatial information and reflect only the preparation to make a reaching movement to the next target to appear. Several lines of evidence were presented by Calton et al. (2002) supporting the nonspatial alternative. The current data provide another opportunity to address this point.

Consider, on the one hand, what we would see if delay activity reflects the animal's prediction of where the target will appear. In this scenario, high firing reflects an expectation that the target will appear in the response field, and low firing reflects an expectation that the target will appear outside the field. Reaction times should be fastest when the animal correctly anticipates the location of the target (Posner, 1980; Bowman et al., 1993). As a result, high delay activity should be associated with short RTs only when the target in fact appears in the response field but should be associated with long RTs when the target appears unexpectedly outside the response field. The reverse would hold for low delay activity: we predict short RTs when the target lands outside the field but long RTs when the target lands inside. Thus, if delay activity reflects the animal's expectation of where the target will appear, then the correlation between delay activity and RT should be negative for target-in trials but positive for target-out trials.

On the other hand, if effector-specific delay period activity is independent of spatial information, then by definition we would predict that the correlation between delay activity and RT would be independent of target location.

The results are very clear. The average correlation coefficient for reach trials on which the target appeared in the response field was $-0.06(p=0.05)$. The average correlation coefficient for trials on which the target was outside the field was -0.08 ( $p=$ 0.003 ). When only cells with greater delay activity on reach compared with saccade trials are considered (Calton et al., 2002), correlation coefficients of -0.16 and -0.13 are obtained for preferred and null trials, respectively. These findings provide strong evidence against the idea that delay activity reflects spatial target prediction and strong support for the alternative hypothesis that the activity reflects a signal that is independent of spatial information.

There is yet another possible explanation for the correlation between delay activity and reach RTs. Delay period activity in PRR tends to ramp upward with time (Fig. 1B). Reach RTs decrease with longer delay periods (see above, Behavior). It is conceivable that these two observations have no causal relationship and yet account for the correlation we observed. Evidence against this supposition includes the fact that the effect of delay period duration on RT was twice as large in M2 as in M1, yet the neuronal-behavioral correlation was better for M1 than M2 (correlation coefficients of -0.09 vs -0.04 , respectively). A more direct test is to ask whether a correlation remains when data are detrended by subtracting the mean values for PRR activity and RT for each delay period duration and then recombining and reanalyzing the mean-subtracted data. This approach yields a correlation of $-0.06(p=0.015)$. When only the 45 cells with significantly higher delay period activity on reach compared with saccade trials are considered, the correlation is increased to -0.12 $(p=0.004)$. These results clearly rule out the possibility that the correlations reflect unrelated trends in reach RT and delay activity over time.

\section{Target-delay-cue and memory tasks}

Surprisingly, there was no significant correlation $(p>0.05)$ between PRR delay activity and movement RT in each of the targetdelay-cue and standard memory tasks (Table 3). The lack of a significant effect also applied to subpopulations of neurons with effector-specific differences in delay activity (last two columns) and to visual, visuomovement, and movement subpopulations from the two regions (data not shown). Even when data from trials with targets appearing inside and outside of the response field were analyzed separately, no significant correlations were obtained (data not shown). Finally, in area 5 exclusive of PRR, there were no significant effects for any of the three tasks (data not shown). 
Table 3. Mean correlation coefficient $(r)$ between delay period firing rate and RT

\begin{tabular}{|c|c|c|c|c|c|c|c|}
\hline \multirow[b]{2}{*}{ Area } & \multirow[b]{2}{*}{ Task } & \multirow[b]{2}{*}{ Cells } & \multirow[b]{2}{*}{ Effector } & \multicolumn{2}{|l|}{ All cells } & \multicolumn{2}{|c|}{ Effector-specific cells } \\
\hline & & & & $r$ & $p$ value & $r$ & $p$ value \\
\hline \multirow[t]{6}{*}{ PRR } & \multirow[t]{2}{*}{ Cue-delay-target } & \multirow[t]{2}{*}{$138(76)$} & Reach & -0.07 & 0.001 & -0.15 & $<0.001$ \\
\hline & & & Saccade & 0.00 & 0.99 & 0.04 & 0.37 \\
\hline & \multirow[t]{2}{*}{ Target-delay-cue } & \multirow[t]{2}{*}{$138(76)$} & Reach & -0.01 & 0.31 & -0.04 & 0.15 \\
\hline & & & Saccade & -0.02 & 0.44 & -0.04 & 0.25 \\
\hline & \multirow[t]{2}{*}{ Memory task } & \multirow[t]{2}{*}{$67(0)$} & Reach & 0.06 & 0.19 & 0.06 & 0.50 \\
\hline & & & Saccade & -0.03 & 0.34 & -0.08 & 0.23 \\
\hline \multirow[t]{6}{*}{ Area 5} & \multirow[t]{2}{*}{ Cue-delay-target } & \multirow[t]{2}{*}{$75(18)$} & Reach & -0.03 & 0.18 & -0.03 & 0.52 \\
\hline & & & Saccade & 0.04 & 0.28 & -0.07 & 0.39 \\
\hline & \multirow[t]{2}{*}{ Target-delay-cue } & \multirow[t]{2}{*}{$75(18)$} & Reach & -0.01 & 0.39 & -0.02 & 0.61 \\
\hline & & & Saccade & 0.00 & 0.90 & -0.06 & 0.38 \\
\hline & \multirow[t]{2}{*}{ Memory task } & \multirow[t]{2}{*}{$50(0)$} & Reach & 0.02 & 0.45 & 0.00 & 0.92 \\
\hline & & & Saccade & 0.01 & 0.82 & 0.07 & 0.24 \\
\hline
\end{tabular}

Data for cue-delay-target task are for movements in either the preferred or null direction; data for the target-delay-cue and memory tasks are for the preferred direction only. $p$ values are not corrected for multiple tests. The value in the 'Cells' column is the number of cells tested in that particular paradigm using isolated eye movements and isolated arm movements. There follows, in parentheses, the subset of cells that were also tested using combined eye and arm movements. 'Effector-specific cells' include 33\% $(n=45)$ and $24 \%(n=18)$ of cells, respectively, from PRR and from area 5 exclusive of PRR, which showed significantly greater activity when a reach compared with a saccade was planned $[10 \%(n=7)$ and $5 \%(n=7)$ of cells in PRR and area 5 , respectively, showed the reverse effect: significantly greater activity when a saccade compared with a reach was planned].

\section{Discussion}

Our results show a specific correlation between reach reaction time and neural activity during a delay period in the parietal reach region, before the specification of the target for the movement. Correlation coefficients were low $(-0.07)$, but the effect was consistent across two animals and highly significant. A type II regression suggests that, with each additional spike/s of activity occurring immediately before target presentation, reach RT decreases by $24.9 \mathrm{~ms}$. This correlation was highly effector specific; there was no effect of PRR delay period activity on saccade RT.

The current results are noteworthy for three reasons. First, they concern the posterior parietal cortex, a region often viewed as subserving processes far removed from motor output. Most previous studies of correlations between neural activity and RT were performed in frontal cortex, basal ganglia, or brainstem (see Introduction). Second, the correlations that we report are not confined to a particular direction of movement. Most previous studies find correlations for movements made in one particular direction and no correlation or an anticorrelation for movements in the opposite direction (Cohen et al., 2004). In PRR, we find correlations with movements both into and out of the response field of the cell. This is indicative of a nonspatial effect and, in particular, an effect that is not related to spatial prediction. This is consistent with the finding that neural correlates of spatial prediction in an eye movement task are not found in the posterior parietal cortex (Lawrence and Snyder, 2006). Third, the absence of a correlation between activity and saccadic RT indicates that the effect of neural activity is specific to reaching movements and not simply a result of generic processes (e.g., nonspatial attention to the task, arousal, vigilance). (Surprisingly, the lack of a correlation between neuronal activity and saccadic RT held even for saccades that accompanied reaching movements.) Together, these data suggest that PRR plays a significant and causal role in the generation of visually guided reaching movements of the upper limbs.

A specific relationship between delay period activity and reach RT occurred only when there was spatial uncertainty. For tasks in which there was spatial certainty (the standard memory task and the target-delay-cue task), spatial information was encoded by PRR, but the level of activity was unrelated to reach RT. At first pass, this seems illogical. If, as suggested previously, the role of PRR is to convey spatial information for visually guided reaches
(Snyder et al., 1997; Calton et al., 2002), then one might expect that a more robust sustained spatial response would result in a shorter RT. How can the lack of an activity-RT correlation in standard memory and target-delay-cue tasks be explained?

In fact, our findings are consistent with a critical and effector-specific role of PRR in processing spatial information for an upcoming reach. While awaiting the target in the cue-delay-target task, the animal knows the type of movement to make but lacks spatial information. If this spatial information must pass through the dorsal visual stream, and in particular, through PRR, to drive an arm movement, then anything that influences the speed of the PRR response will affect RT. When a spatial target appears inside the response field of a cell, that cell receives an incoming volley of activity. If this incoming volley adds to the existing activity, then any given rate of firing will be achieved more rapidly given an elevated baseline. This in turn will likely to lead to a faster reaching movement. The key point is that the speed with which the animal moves depends on how fast spatial information is propagated from the visual system to the arm muscles, and so elevated levels of firing in areas that lie along that pathway are likely to lead to faster reaches.

In contrast, consider the standard delayed reach task. Here, reach $\mathrm{RT}$ depends on how fast the subject can respond to a trigger signal, that is, fixation light offset. During the delay, the target location is represented in PRR and in multiple other brain areas. These representations must be blocked from influencing the arm muscles (Kalaska et al., 1995). Once the fixation point is extinguished, a representation of that event must release this blockade. As a result, a major determinant of reach RT will be how long it takes to process fixation point offset. Because the fixation point is not represented by PRR neurons with peripheral response fields, this process will be independent of PRR activity. In other words, we suggest that it is the speed with which the gate is opened, and not the amplitude of the robust signal "waiting behind" that gate, that determines reach RT in the standard delayed reach task.

The same argument applies to the target-delay-cue task. Here, a change in color at the fixation point serves to trigger a saccade or a reach. Once again, RT does not depend on the precise level of PRR activity when the spatial information is provided long before the trigger that releases the movement and when the trigger itself is not processed in PRR. This is in contrast to the situation in the cue-delay-target task, in which the spatial information processed in PRR is the trigger that releases the movement.

Thus, our data are consistent with the idea that PRR is instrumental in conveying spatial information to downstream structures involved in initiating visually guided reaching movements. A subset of PRR cells receive a "boost" when an animal plans in advance to make a reaching movement (Calton et al., 2002), and this extra activity appears to result in faster reach reaction times. The idea that PRR may be limited to playing a role only in visually guided movements is supported by functional imaging work in humans showing no preparatory responses in the parietal cortex in conjunction with a nonspatial, nonvisually guided fingertapping task (Thoenissen et al., 2002). These results suggest that PRR might best be thought of not as encoding a particular psy- 
chological function, such as motor intention or motor set, but rather as being a part of the machinery by which visual information is processed and conveyed to downstream areas for the purposes of aiming a reach.

We argued previously that the elevated delay period activity in PRR during a reach trial compared with a saccade trial is evidence that PRR receives not only spatial information but also effector information. It is tempting to try to simplify this description by viewing delay activity as representing a previous probability, in the Bayesian sense, that a spatial target is about to appear. The utility of this description is that it appears to eliminate the need for an effector input into PRR. However, the fact that delay period activity is elevated on reach but not saccade trials demonstrates that a simple spatial interpretation is incorrect. Of course, this does not invalidate the Bayesian approach to delay activity. Instead, it shows that the probability that is being represented is either that a reach will occur or that a spatial target for a reach will appear.

Trial-to-trial variations in firing rate are likely to be shared, to some extent, across cells (Shadlen et al., 1996). Without such correlated firing, the contribution of any one neuron to any one behavioral output would be extremely small: no larger than one over the number of neurons involved in that behavior. Our data show that each PRR neuron contributes at most $\sim 1 \%$ of the variance in $\mathrm{RT}$ firing. This is consistent with a pool of $\sim 100$ uncorrelated neurons whose firing rate determines RT. It is also consistent with a much larger pool of correlated neurons whose firing rate influences RT. The fact that delay activity is correlated with reach RT even for reaches outside the receptive field is strong evidence that neuronal firing is correlated, because there is otherwise no reason to expect that delay activity would show similar correlations with reach RTs both into and out of the response field of the neuron.

Many previous studies demonstrated a connection between area 5 and arm movements (Seal et al., 1982; Crammond and Kalaska, 1989). This makes our basic finding, that PRR activity is related to reach RT whereas area 5 activity is not, somewhat surprising. Area 5 may in fact show a stronger relationship with reach dynamics at the time of the movement. We also cannot rule out the possibility that portions of area 5 in which we did not record may show effects more similar to those found in PRR.

Finally, it is conceivable that the relationship we see between activity and RT derives from somatosensory cortex, propagated through area 5 to PRR (Nishihira et al., 1987). Animals might co-contract their arm muscles when preparing a reach, perhaps particularly on trials in which the ultimate direction of that reach remains unknown. Co-contraction could be encoded in the afferents, and this signal might then be passed on to PRR neurons. The magnitude of co-contraction might be related to the degree of preparation, resulting in the correlation between activity and reach RT. However, we found that cells in area 5 outside of PRR did not show any relationship between firing rate and reach or saccade RT, making this explanation less likely. Furthermore, previous studies of go/no-go paradigms found no evidence for anticipatory co-contraction (Kalaska and Crammond, 1995). Instead, we believe that the most likely explanation for our findings is that increased PRR firing during the cue-delay-target task reflects task-specific preparatory processing, perhaps analogous to the preparatory task-specific activity that has been found in the parietal cortex during the preparation of complex cognitive tasks (Stoet and Snyder, 2004).

\section{References}

Alexander GE, Crutcher MD (1990) Preparation for movement: neural representations of intended direction in three motor areas of the monkey. J Neurophysiol 64:133-150.

Andersen RA (1995) Encoding of intention and spatial location in the posterior parietal cortex. Cereb Cortex 5:457-469.

Baddeley A (2003) Working memory: looking back and looking forward. Nat Rev Neurosci 4:829-839.

Basso MA, Wurtz RH (1997) Modulation of neuronal activity by target uncertainty. Nature 389:66-69.

Basso MA, Wurtz RH (1998) Modulation of neuronal activity in superior colliculus by changes in target probability. J Neurosci 18:7519-7534.

Bisley JW, Goldberg ME (2003) Neuronal activity in the lateral intraparietal area and spatial attention. Science 299:81-86.

Bowman EM, Brown VJ, Kertzman C, Schwarz U, Robinson DL (1993) Covert orienting of attention in macaques. I. Effects of behavioral context. J Neurophysiol 70:431-443.

Bruce CJ, Goldberg ME (1985) Primate frontal eye fields. I. Single neurons discharging before saccades. J Neurophysiol 53:603-635.

Bushnell MC, Goldberg ME, Robinson DL (1981) Behavioral enhancement of visual responses in monkey cerebral cortex. I. Modulation in posterior parietal cortex related to selective visual attention. J Neurophysiol 46:755-772.

Calton JL, Dickinson AR, Snyder LH (2002) Non-spatial, motor-specific activation in posterior parietal cortex. Nat Neurosci 5:580-588.

Churchland MM, Yu BM, Ryu SI, Santhanam G, Shenoy KV (2006) Neural variability in premotor cortex provides a signature of motor preparation. J Neurosci 26:3697-3712.

Cohen YE, Cohen IS, Gifford III GW (2004) Modulation of LIP activity by predictive auditory and visual cues. Cereb Cortex 14:1287-1301.

Connolly JD, Goodale MA, Menon RS, Munoz DP (2002) Human fMRI evidence for the neural correlates of preparatory set. Nat Neurosci 5:1345-1352.

Connolly JD, Andersen RA, Goodale MA (2003) FMRI evidence for a "parietal reach region" in the human brain. Exp Brain Res 153:140-145.

Connolly JD, Goodale MA, Goltz HC, Munoz DP (2005) FMRI activation in the human frontal eye field is correlated with saccadic reaction time. J Neurophysiol 94:605-611.

Constantinidis C, Steinmetz MA (2001) Neuronal responses in area 7a to multiple-stimulus displays: I Neurons encode the location of the salient stimulus. Cereb Cortex 11:581-591.

Corbetta M, Shulman GL (2002) Control of goal-directed and stimulusdriven attention in the brain. Nat Rev Neurosci 3:201-215.

Corbetta M, Miezin FM, Shulman GL, Petersen SE (1993) A PET study of visuospatial attention. J Neurosci 13:1202-1226.

Crammond DJ, Kalaska JF (1989) Neuronal activity in primate parietal cortex area 5 varies with intended movement direction during an instructeddelay period. Exp Brain Res 76:458-462.

Crammond DJ, Kalaska JF (2000) Prior information in motor and premotor cortex: activity during the delay period and effect on pre-movement activity. J Neurophysiol 84:986-1005.

DeSouza JF, Menon RS, Everling S (2003) Preparatory set associated with pro-saccades and anti-saccades in humans investigated with event-related FMRI. J Neurophysiol 89:1016-1023.

Dickinson AR, Calton JL, Snyder LH (2003) Nonspatial saccade-specific activation in area LIP of monkey parietal cortex. J Neurophysiol 90:2460-2464.

Dorris MC, Munoz DP (1998) Saccadic probability influences motor preparation signals and time to saccadic initiation. J Neurosci 18:7015-7026.

Dorris MC, Pare M, Munoz DP (1997) Neuronal activity in monkey superior colliculus related to the initiation of saccadic eye movements. J Neurosci 17:8566-8579.

Elkington PT, Kerr GK, Stein JS (1992) The effect of electromagnetic stimulation of the posterior parietal cortex on eye movements. Eye 6:510-514.

Everling S, Munoz DP (2000) Neuronal correlates for preparatory set associated with pro-saccades and anti-saccades in the primate frontal eye field. J Neurosci 20:387-400.

Everling S, Dorris MC, Klein RM, Munoz DP (1999) Role of primate superior colliculus in preparation and execution of anti-saccades and prosaccades. J Neurosci 19:2740-2754.

Fujii N, Mushiake H, Tanji J (2002) Distribution of eye- and arm- 
movement-related neuronal activity in the SEF and in the SMA and preSMA of monkeys. J Neurophysiol 87:2158-2166.

Hanes DP, Schall JD (1996) Neural control of voluntary movement initiation. Science 274:427-430.

Hanes DP, Thompson KG, Schall JD (1995) Relationship of presaccadic activity in frontal eye field and supplementary eye field to saccade initiation in macaque: Poisson spike train analysis. Exp Brain Res 103:85-96.

Hoshi E, Tanji J (2004) Functional specialization in dorsal and ventral premotor areas. Prog Brain Res 143:507-511.

Jaeger D, Gilman S, Aldridge JW (1993) Primate basal ganglia activity in a precued reaching task: preparation for movement. Exp Brain Res 95:51-64.

Judge SJ, Richmond BJ, Chu FC (1980) Implantation of magnetic search coils for measurement of eye position: an improved method. Vision Res 20:535-538.

Kalaska JF, Crammond DJ (1995) Deciding not to GO: neuronal correlates of response selection in a $\mathrm{GO} / \mathrm{NOGO}$ task in primate premotor and parietal cortex. Cereb Cortex 5:410-428.

Kurata K (1993) Premotor cortex of monkeys: set- and movement-related activity reflecting amplitude and direction of wrist movements. J Neurophysiol 69:187-200.

Lawrence BM, Snyder LH (2006) A comparison of effector-specific signals in frontal and parietal cortices. J Neurophysiol 96:1393-1400.

Lecas JC, Requin JA, Anger C, Vitton N (1986) Changes in neuronal activity of the monkey precentral cortex during preparation for movement. J Neurophysiol 56:1680-1702.

Lee IH, Assad JA (2003) Putaminal activity for simple reactions or selftimed movements. J Neurophysiol 89:2528-2537.

Nishihira Y Araki H, Ishihara A (1987) Changes in central somatosensory pathways accompanying reaction movements. Percept Mot Skills 64:1251-1260.

Posner MI (1980) Orienting of attention. Q J Exp Psychol 32:3-25.

Reddi BA, Asrress KN, Carpenter RH (2003) Accuracy, information, and response time in a saccadic decision task. J Neurophysiol 90:3538-3546.

Riehle A, Requin J (1993) The predictive value for performance speed of preparatory changes in neuronal activity of the monkey motor and premotor cortex. Behav Brain Res 53:35-49.
Requin J, Lecas J-L, Vitton N (1990) A comparison of preparation-related neuronal activity changes in the prefrontal, premotor, primary motor and posterior parietal areas of the monkey cortex: preliminary results. Neurosci Lett 111:151-156.

Roitman JD, Shadlen MN (2002) Response of neurons in the lateral intraparietal area during a combined visual discrimination reaction time task. J Neurosci 22:9475-9489.

Seal J, Gross C, Bioulac B (1982) Activity of neurons in area 5 during a simple arm movement in monkeys before and after deafferentation of the trained limb. Brain Res 250:229-243.

Shadlen MN, Britten KH, Newsome WT, Movshon JA (1996) A computational analysis of the relationship between neuronal and behavioral responses to visual motion. J Neurosci 16:1486-1510.

Sirigu A, Daprati E, Ciancia S, Giraux P, Nighoghossian N, Posada A, Haggard $\mathrm{P}$ (2004) Altered awareness of voluntary action after damage to the parietal cortex. Nat Neurosci 7:80-84.

Snyder LH, Batista AP, Andersen RA (1997) Coding of intention in the posterior parietal cortex. Nature 386:167-170.

Snyder LH, Calton JL, Dickinson AR, Lawrence BM (2002) Eye-hand coordination: saccades are faster when accompanied by a coordinated arm movement. J Neurophysiol 87:2279-2286.

Stoet G, Snyder LH (2003) Task preparation in macaque monkeys (Macaca mulatta). Anim Cogn 6:121-130.

Stoet G, Snyder LH (2004) Single neurons in posterior parietal cortex of monkeys encode cognitive set. Neuron 42:1003-1012.

Tanji J, Evarts EV (1976) Anticipatory activity of motor cortex neurons in relation to direction of an intended movement. J Neurophysiol 39:1062-1068.

Thoenissen D, Zilles K, Toni I (2002) Differential involvement of parietal and precentral regions in movement preparation and motor intention. J Neurosci 22:9024-9034.

Weinrich M, Wise SP, Mauritz KH (1984) A neurophysiological study of the premotor cortex in the rhesus monkey. Brain 107:385-414.

Yantis S, Jonides J (1984) Abrupt visual onsets and selective attention: evidence from visual search. J Exp Psychol Hum Percept Perform 10:601621. 